\title{
Precise Localization of Multiple Noncooperative Objects in a Disordered Cavity by Wave Front Shaping
}

\author{
Philipp del Hougne, ${ }^{1,2, *}$ Mohammadreza F. Imani, ${ }^{2}$ Mathias Fink, ${ }^{1}$ David R. Smith, ${ }^{2}$ and Geoffroy Lerosey ${ }^{3}$ \\ ${ }^{1}$ Institut Langevin, CNRS UMR 7587, ESPCI Paris, PSL Research University, 1 rue Jussieu, 75005 Paris, France \\ ${ }^{2}$ Department of Electrical and Computer Engineering, Center for Metamaterials and Integrated Plasmonics, \\ Duke University, Durham, North Carolina 27708, USA \\ ${ }^{3}$ Greenerwave, ESPCI Paris Incubator PC'up, 6 rue Jean Calvin, 75005 Paris, France
}

(Received 31 January 2018; published 6 August 2018)

\begin{abstract}
Complicated multipath trajectories of waves in disordered cavities cause object localization to be very challenging with traditional ray-tracing approaches. Yet it is known that information about the object position is encoded in the Green's function. After a calibration step, traditional time-reversal approaches retrieve a source's location from a broadband impulse response measurement. Here, we show that a nonemitting object's scattering contribution to a reverberant medium suffices to localize the object. We demonstrate our finding in the microwave domain. Then, we further simplify the scheme by replacing the temporal degrees of freedom (d.o.f.) of the broadband measurement with spatial d.o.f. obtained from wave front shaping. A simple electronically reconfigurable reflectarray inside the cavity dynamically modulates parts of the cavity boundaries, thereby providing spatial d.o.f. The demonstrated ability to localize multiple noncooperative objects with a single-frequency scheme may have important applications for sensors in smart homes.
\end{abstract}

DOI: 10.1103/PhysRevLett.121.063901

Localizing one or multiple objects in a disordered reverberant environment is intrinsically challenging since a wave, for instance, an acoustic wave or a microwave, used to probe the scene will follow many seemingly arbitrary paths involving multiple reflections [1-5]. Yet, indoor localization is a highly sought-after ability for futuristic context-aware environments [6-8]. Prevalently, objects that actively collaborate with the localization task by emitting a signal or carrying a tag are considered [9], such that the problem becomes that of source localization. To localize such a source, the measured wave field is separated into its source component and its reverberated component, as demonstrated, for instance, in acoustics with advanced signal processing techniques [10-12]. Alternatively, rather than "dereverberating" the wave field, the medium's complexity can be exploited because it assigns unique Green's functions to each potential source position; once a dictionary characterizing the environment is established, the measured Green's function only needs to be compared to the dictionary to identify the source location [13-17].

Complex media completely scramble any propagating wave front. Time reversal (TR) has proven to be a powerful

Published by the American Physical Society under the terms of the Creative Commons Attribution 4.0 International license. Further distribution of this work must maintain attribution to the author(s) and the published article's title, journal citation, and DOI. tool that leverages this complexity to achieve spatiotemporal focusing at a selected position by reemitting a timereversed Green's function [18]. The localization schemes based on the correlation of a measured signal with dictionary entries may be interpreted as virtual TR experiments yielding a single maximum value that reveals the object position [13]. Demonstrations range from using finger impacts (source) on a glass plate (reverberant cavity) in acoustics, enabling one to convert any solid object into an interactive touchscreen $[13,18]$, to precisely localizing a microwave emitter in a room [15-17,19]. Various demonstrations elegantly implemented the idea by using channel state information between an access point and a wireless device to localize the latter $[20,21]$. Moreover, understanding that the natural complexity provides useful degrees of freedom (d.o.f.) enabled the conception of manmade complex metamaterials to achieve single-sensor multispeaker listening [22].

More recently, wave front shaping (WFS) emerged as an alternative way of controlling waves in complex media. The idea was initially introduced in optics where a plane wave front is purposefully shaped such that the contributions from different parts interfere constructively at a chosen focal point after propagation through the complex medium [23-26]. The concept has since been transposed to the microwave domain using simple electronically tunable reflectarrays termed spatial microwave modulator (SMM), enabling, for instance, spatiotemporal focusing in a disordered cavity [27,28]. Appealing due to its simple hardware, the WFS of microwaves has found applications ranging from 
wireless indoor communication to security screening and energy harvesting [29-32].

In this Letter, we first suggest that leveraging a medium's complexity for localization does not require the object to actively emit. Indeed, in line with current trends in devicefree indoor localization using Wi-Fi or ambient signals $[33,34]$, we consider theoretically and demonstrate with a microwave experiment that the scattering contribution of one or multiple objects to a reverberant medium suffices to retrieve the object positions. This concept then permits us to make the transition towards a single-frequency wave front shaping scheme for localization, in which the broadband Green's function measurement is replaced with random modulations of the cavity's boundary conditions.

First, we discuss the theoretical background underlying both our frequency diverse and WFS approaches. Second, we outline our setup and analyze how unique the measured dictionary entries are. Third, we run a series of tests with up to three objects to demonstrate the feasibility of both proposed approaches. Finally, we address the question of how many d.o.f. are needed to guarantee a successful localization.

Our suggestion to localize nonemitting objects using their scattering contribution is inspired by works on diffusing reverberant acoustic wave spectroscopy (DRAWS) [35-37]. DRAWS considers the case of weak scatterers moving in a cavity, assuming (i) the cavity is sufficiently reverberant to create a diffuse wave field, and (ii) the mean free path between scattering events is large compared to the cavity dimensions. These constraints match a variety of real-life scenarios ranging from fish in an aquarium, via mice in an open field test, to humans in a large room [35,38,39]. While DRAWS links the wave field's autocorrelation to characteristics of the scatterers (quantity, scattering cross section), here we borrow the assumption that under the stated conditions, the interplay of multiple scattering off the objects and multiple reflections off the cavity walls can be unraveled [40]. Then, the Green's function $S(f)$ between two antennas arbitrarily placed inside the cavity may be decomposed as

$$
S(f)=s_{\text {cav }}(f)+\sum_{\text {scatterers }} s_{i}(f),
$$

where $s_{\text {cav }}(f)$ and $s_{i}(f)$ are the contributions to the Green's function by the static cavity and scatterer $i$, respectively.

We consider the contribution $s_{i}$ of an object at position $i$ to the Green's function as its unique signature. Essentially, the scatterer acts as a secondary source. The dictionary D contains one entry per potential object position per potential object type (different size, shape, or material). First, $s_{\text {cav }}$ is measured in the empty cavity. Then, for each dictionary entry in turn, the corresponding object is placed at the corresponding position, the transmitted field $S_{i}$ is measured, and $s_{i}$ is estimated as $S_{i}-s_{\text {cav }}$. Dealing with multiple identical objects does not require a prohibitively large D; the ability to unravel different scattering contributions with Eq. (1) enables maintaining the same $\mathbf{D}$ as if there was only one object of each type. Sufficiently different objects can be localized and identified due to the object-type-dependent signature. The principle is not limited to reflecting objects since absorption also leaves a signature on the Green's function by suppressing paths [41]. In cases of known room geometry, $\mathbf{D}$ can also be estimated by means of numerical simulation instead of tedious measurements [42-44]. As discussed in Ref. [13], the resolution is subject to the Rayleigh limit: two positions $i$ and $j$ should be separated by at least half the central operating wavelength $\lambda_{0}$.

The broadband Green's function measurement provides a certain number of independent probes of the cavity wave field. This number of temporal d.o.f. is given by

$$
N_{t}=\Delta f / \Delta f_{\text {corr }},
$$

where $\Delta f$ is the range over which the Green's function is measured, and $\Delta f_{\text {corr }}$ is the correlation frequency $[45,46]$. The latter is the ratio between central frequency $f_{0}$ and the cavity's quality factor $Q, \Delta f_{\text {corr }}=f_{0} / Q$, and corresponds to the minimum separation between two frequencies required for the corresponding fields to be independent. The temporal d.o.f. may be replaced by spatial d.o.f. This enables single-frequency operation, avoiding spectrum allotment concerns and simplifying the hardware. A naive way would be to place $N_{s}$ antennas at random independent locations inside the cavity, separated by at least $\lambda_{0} / 2$, and measure the field at a single frequency at each of them.

A more elegant way is found in the realm of wave front shaping by placing a SMM in the environment under test. Here, we use random rather than carefully optimized SMM configurations; each random configuration yields a completely different wave field, such that the number of random SMM configurations used to probe the cavity constitutes as many spatial d.o.f. We thus replace the broadband dictionary entries $s_{i}(f)$ by $s_{i}(m)$ obtained with WFS, where $m$ denotes the $m$ th random SMM configuration.

Very recently, dynamic cavity boundaries have also been introduced in biomedical microwave imaging to change the interrogating field inside metallic chambers $[47,48]$ and then reconstruct the sample's composition numerically given broadband transmission measurements. Such a combination of spatial and temporal d.o.f. is, of course, also feasible for the localization scheme we present, but it is not studied here. Computational imaging with dynamic metasurface apertures (DMAs) enables single-frequency operation in a similar spirit [31,49]. DMAs placed inside a cavity enable one to tune the antenna coupling to the cavity modes and, thus, also probe the wave field in several independent ways, as recently demonstrated for "smart" motion detection [50]. This may constitute an alternative implementation of the localization by WFS presented here.

For our experiments, we use a disordered, metallic cavity $\left(1.1 \mathrm{~m}^{3}, Q=1034\right)$ in which we place up to three identical 


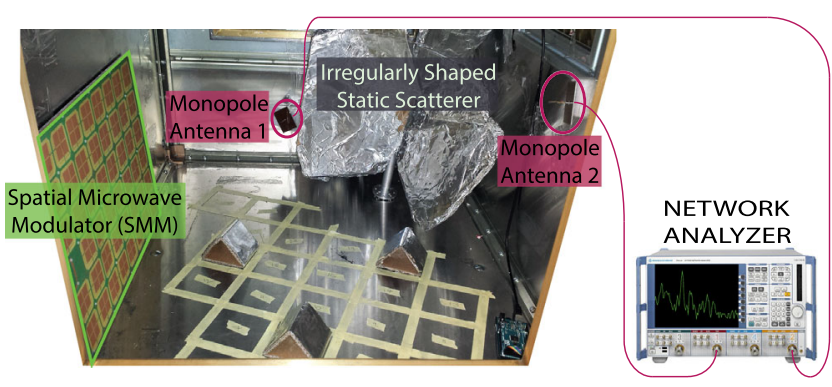

FIG. 1. Experimental setup. See the main text for a description.

metallic objects (base $9 \times 9 \mathrm{~cm}^{2}$, height $6.5 \mathrm{~cm}$ ) at positions selected from $\mathcal{P}=23$ predefined possible ones, as shown in Fig. 1. With a network analyzer, we measure the transmitted field between two arbitrarily placed monopole antennas in the interval $2 \mathrm{GHz}<f<3 \mathrm{GHz}$ such that the object size is roughly half a wavelength. A SMM [51] consisting of 39 elements covers about 3\% of the cavity surface. Each element's reflection coefficient can be switched dynamically to mimic Dirichlet or Neumann boundary conditions. The SMM is designed to work around 2.65 GHz. Depending on whether we use temporal or spatial d.o.f., we measure $S\left(f, m_{0}\right)$ or $S\left(f_{0}, m\right)$, fixing either a single random SMM mask $m_{0}$ or working frequency $f_{0}$, respectively.

To begin with, we examine how unique the dictionary entries $s_{i}$ are. The amplitudes of three sample entries are plotted for both the frequency diverse and the WFS approaches in Figs. 2(a) and 2(b); they are clearly distinct by inspection. To rigorously compare two complex valued vectors $s_{i}$ and $s_{j}$, the standard procedure is to calculate the correlation $C_{i, j}=\left|s_{i}^{*} s_{j}\right| / \sqrt{\left|s_{i}^{*}\right|^{2}\left|s_{j}\right|^{2}}$. Alternatively, we can remove the mean of the signatures before comparing them: $C_{i, j}^{\prime}=\left|s_{i}^{\prime *} s_{j}^{\prime}\right| / \sqrt{\left|s_{i}^{*}\right|^{2}\left|s_{j}^{\prime}\right|^{2}}$, with $s_{i}^{\prime}=s_{i}-\left\langle s_{i}\right\rangle$. We define the average similarity of the dictionary entries, $\mathcal{S}$ or $\mathcal{S}^{\prime}$, as the average of the off-diagonal elements of $C$ or $C^{\prime}$. In Figs. 2(c) and 2(d), we contrast the two similarity measures and show their sensitivity to the choice of $m_{0}$ or $f_{0}$, respectively, for frequency diverse or WFS operation.

For the frequency diverse case, as expected, we observe no notable dependence on the chosen random SMM mask $m_{0}$ in Fig. 2(c); $\mathcal{S}=\mathcal{S}^{\prime}$ since $\left\langle s_{i}\right\rangle=0$, as the right panel in Fig. 2(a) reveals. The average similarity value of 0.22 is comparable to what was achieved in Ref. [22] and very well below unity, indicating that the signatures are unique and well suited for the localization task. The sample plots of two $C^{\prime}$ matrices confirm that the signatures are uniformly uncorrelated.

With WFS, there is, of course, a dependence on the working frequency in Fig. 2(d) due to the SMM's limited operation bandwidth. Quite strikingly, however, $\mathcal{S}^{\prime}$ yields substantially more favorable results than $\mathcal{S}$. This can be understood with traditional WFS tools. The Green's
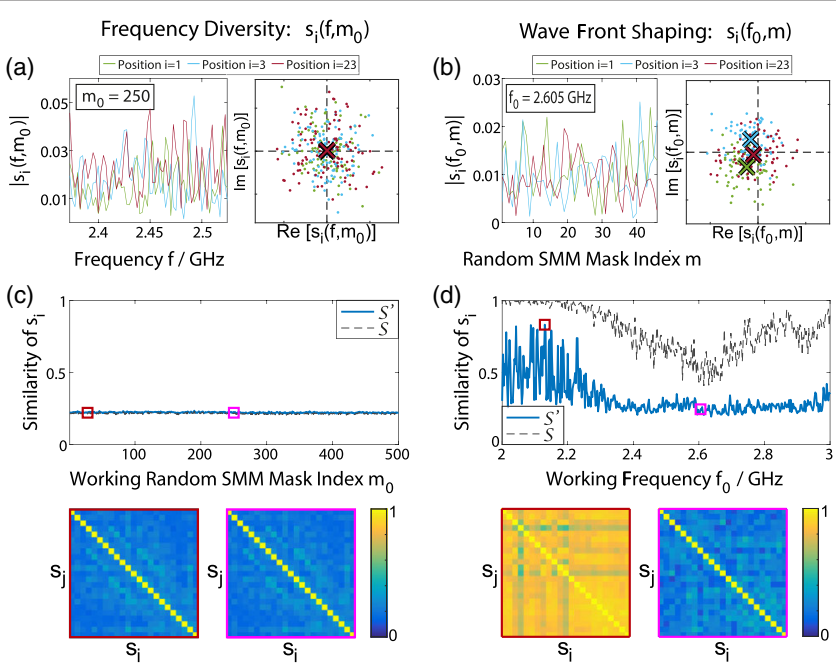

FIG. 2. Uniqueness of dictionary entries using 391 temporal (left) or 500 spatial (right) d.o.f. (a),(b) Magnitude and complex representation of three sample dictionary entries $s_{i}$; the crosses indicate $\left\langle s_{i}\right\rangle$. (c), (d) Average similarity between dictionary entries evaluated in two different ways; cf. main text. Sample similarity matrices $C^{\prime}$ are shown for the indicated cases.

function at $f_{0}$ is the sum of contributions from some $\mathcal{N}$ cavity modes overlapping at $f_{0}$ due to their finite linewidths [52-55]. On average, the SMM may be modeled to provide a phase-binary control over $n$ modes [27]. Here, $n<\mathcal{N}$ due to the small SMM (see Ref. [56]) such that the contributions to the Green's function of some modes are not altered by different SMM configurations. Therefore, the clouds of $s_{i}$ are not centered on the origin of the complex plane in Fig. 2(b): each entry of $s_{i}$ is the sum of a constant and several random phasors. Subtracting $\left\langle s_{i}\right\rangle$ eliminates the contributions from the $\mathcal{N}-n$ uncontrolled modes, carving out the distinct features of the WFS dictionary. Over a $0.5 \mathrm{GHz}$ interval around $2.65 \mathrm{GHz}$, WFS achieves an average similarity coefficient $\mathcal{S}^{\prime}$ of 0.25 , going as low as 0.19 at some $f_{0}$. The sample full $C^{\prime}$ matrices confirm once again that $s_{i}^{\prime}$ are uniformly uncorrelated for $f_{0}$ within said interval, but other $f_{0}$ are almost not modulated by the SMM, yielding heavy correlations.

Having confirmed that an object's contribution $s_{i}^{\prime}$ to the total transmission between two antennas is unique for each object position $i$, both in the frequency diverse and the WFS approaches, we now test the localization scheme experimentally. We place $p \in\{1,2,3\}$ identical objects at random positions chosen from the predefined $\mathcal{P}=23$ ones. We then measure the transmission $S_{\text {meas }}$ between the two antennas and look for the linear combination $s_{\text {synth }}(z)=\sum_{k=1}^{p} s_{i(z, k)}$, where $z$ refers to the combination under consideration, that most closely resembles $s_{\text {meas }}=$ $S_{\text {meas }}-s_{\text {cav }}$, according to the $\mathcal{S}^{\prime}$ criterion (see Ref. [56]). Here, we compute $s_{\text {synth }}$ for all possible permutations of object locations to identify the best one. In principle, the sparse occupation of the predefined positions makes the 
(a) $p=1$ object
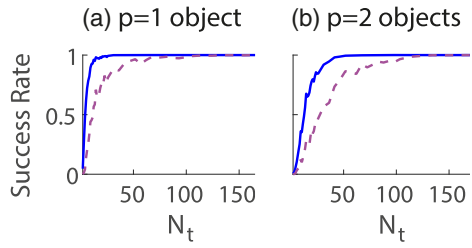

FIG. 3. Average localization success rate using frequency diversity as a function of the number of temporal d.o.f. $N_{t}=$ $\Delta f / \Delta f_{\text {corr }}$ used [see Eq. (2)].

problem suitable for the compressive sensing framework [57], and larger or more complicated systems may prefer more advanced optimization algorithms, such as the one used in Refs. [22,58]. We experimentally test our localization scheme for 20 random permutations of object positions for each value of $p$.

The average localization success rate [56] as a function of the available temporal d.o.f. using the frequency diverse approach is plotted in Fig. 3. We define a success as correctly identifying all $p$ positions. A reconstruction given the exact number of objects inside the cavity (KnowX) is contrasted with one only given the maximum possible number of objects (UpToX). As expected, fewer d.o.f. are needed to achieve a unity success rate knowing the exact number of objects.

The corresponding WFS results in Figs. 4(a)-4(c) unveil which frequencies are particularly well modulated by the SMM: the main band around $2.65 \mathrm{GHz}$ but also two sidebands at 2.4 and $2.9 \mathrm{GHz}$ which arise due to the SMM's working principle of hybridizing resonances outlined in Ref. [51]. Other frequencies are modulated less efficiently but still enough to localize two objects. The curves in Figs. 3(a)-3(c) and Figs. 4(d)-4(f), the latter being the average over the 16 best working frequencies for WFS, are almost identical, confirming the equivalence of the frequency diverse (temporal) and WFS (spatial) d.o.f. Note that in all cases, the minimum number of d.o.f. $\mathcal{I}_{\text {min }}$

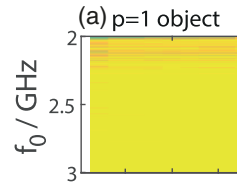

(d)

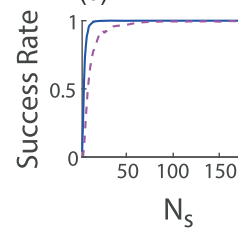

(e)

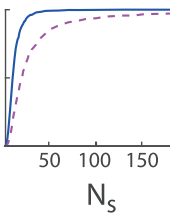

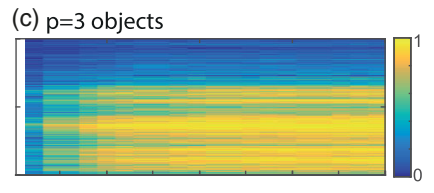

(f)

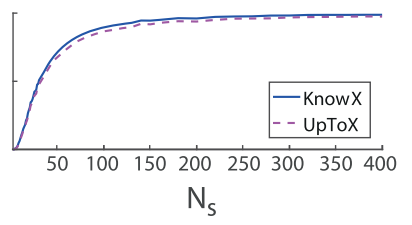

FIG. 4. Average localization success rate using wave front shaping. (a)-(c) Dependence of the success rate on the working frequency for scenarios of known $p$. (d)-(f) Average over the 16 best working frequencies as a function of the number of random SMM configurations $N_{s}$ used.

required to ensure a unity success rate is significantly lower than the number of possible combinations. We define a compression ratio

$$
K=\frac{\mathcal{P} ! /(p !(\mathcal{P}-p) !)}{\mathcal{I}_{\min }},
$$

where the numerator is the number of ways to select $p$ different positions out of the $\mathcal{P}$ possible predefined ones. Table I summarizes the achieved compression results for known $p$.

The compression ratio is above unity in all cases, comparable to the one achieved in Ref. [22] for $p=2$. The utility of "complexity" for compressive sensing is well known from imaging where it was studied in the microwave, terahertz, and optical domains, be it with carefully engineered structured or random illumination, or exploiting complex media as analogue randomizers [59-63].

The question that arises in view of the presented results is: How many d.o.f., regardless of their nature, are required to achieve a unity success rate in localizing $p$ objects? A simple thought experiment reveals that the crucial parameter is the measurement precision. A single d.o.f. with perfect precision would be enough, in principle, since $s_{\text {meas }}$ is never exactly identical for different object positions. In reality, of course, this is not the case: noise or measurement uncertainties can exceed the potentially tiny differences, making them undetectable.

For the case of a single object at one out of $\mathcal{P}$ predefined and characterized positions, a simple toy model can be drafted. Say we have $N$ d.o.f., and the measurement of each of them can take one out of $b$ equiprobable values. For instance, if we have only access to phase measurements, the measurement uncertainty would be $2 \pi / b$. How probable is it to select $\mathcal{P}$ distinct signatures out of the $b^{N}$ possible ones? The answer to this well-known combinatorial problem is

$$
\prod_{j=1}^{\mathcal{P}} \frac{b^{N}-j+1}{b^{N}}=\frac{b^{N} !}{b^{N \mathcal{P}}\left(b^{N}-\mathcal{P}\right) !} .
$$

To ensure unique signatures, we need this probability to reach unity. Our obtained values of $\mathcal{I}_{\min }$ most closely resemble the case of $b=2$ in this idealistic toy model. As our network analyzer's dynamic range is certainly larger than that and the noise level in our cavity is very low, this can be attributed mainly to imprecise positioning of the

TABLE I. Compression ratios with known $p$. Superscripts denote the use of temporal $(t)$ or spatial $(s)$ d.o.f.

\begin{tabular}{lcrrcc}
\hline \hline$p$ & $\mathcal{P} ! /(p !(\mathcal{P}-p) !)$ & $\mathcal{I}_{\min }^{t}$ & $\mathcal{I}_{\min }^{s}$ & $K^{t}$ & $K^{s}$ \\
\hline 1 & 23 & 15 & 14 & $1.5: 1$ & $1.6: 1$ \\
2 & 253 & 47 & 45 & $5.4: 1$ & $5.6: 1$ \\
3 & 1771 & 232 & 220 & $7.6: 1$ & $8.1: 1$ \\
\hline \hline
\end{tabular}


object on the predefined positions. Extending the toy model to cases with $p>1$ is mathematically less straightforward, but we simulated the scenario numerically (see Ref. [56]). With $b=2$ and $\mathcal{P}=23$, the toy model predicts a need of $N=20$ d.o.f. to localize $p=2$ objects. This is about half of what we needed experimentally; cf. Table I. Two additional effects become important for $p>1$. First, the two objects are not perfectly identical to the one used to establish the dictionary. Second, the linear correlation-free nature of Eq. (1) is only a first order approximation.

To conclude, in this Letter, we started off by showing that the uniqueness of Green's functions for different object positions inside a disordered cavity can be exploited for object localization without requiring the object to collaborate. We experimentally proved that the object's contribution to a transmission measurement between two simple antennas at arbitrary positions inside the cavity can serve as a unique signature. Then, we proposed and experimentally demonstrated that the temporal d.o.f. of such a broadband transmission measurement may be elegantly replaced with spatial d.o.f. obtained by wave front shaping. We successfully identified the positions of up to three objects, using up to 8 times fewer measurements than there were potential object permutations. Finally, we clarified the importance of the measurement precision as key factor in determining how many d.o.f. a realistic scheme requires.

Having removed the need for object collaboration and that for broadband operation, our proof of concept can be envisioned as the basis for futuristic localization or tracking sensors in smart homes [64], possibly in combination with existing Wi-Fi infrastructure. The presence of more losses in real life would extend the range of validity of Eq. (1) and not affect the amount of available spatial d.o.f. from WFS but could potentially deteriorate the measurements' dynamic range such that a few additional d.o.f. would be needed (see Ref. [56]). Scaled-up versions could benefit from a tailored compressive sensing algorithm and potentially learning algorithms such as recurrent neural networks to deal with evolving room or object shapes $[65,66]$. Transmission measurements at $\mathrm{MHz}$ rates are within reach with improved SMM electronics and a custom singlefrequency radio $[67,68]$.

P. d. H. thanks Julien de Rosny for fruitful discussions and acknowledges funding from the French "Ministère de la Défense, Direction Générale de l'Armement." The raw data are available in Ref. [69].

The project was initiated, conceptualized, conducted, and written up by P.d.H. All authors contributed with thorough discussions.

*philipp.delhougne@gmail.com

[1] E. Bogomolny, P. Leboeuf, and C. Schmit, Phys. Rev. Lett. 85, 2486 (2000).
[2] D. Laurent, O. Legrand, and F. Mortessagne, Phys. Rev. E 74, 046219 (2006).

[3] T. Tudorovskiy, U. Kuhl, and H.-J. Stöckmann, New J. Phys. 12, 123021 (2010).

[4] J.-B. Gros, U. Kuhl, O. Legrand, F. Mortessagne, E. Richalot, and D. V. Savin, Phys. Rev. Lett. 113, 224101 (2014).

[5] X. Li, Z. D. Deng, L. T. Rauchenstein, and T. J. Carlson, Rev. Sci. Instrum. 87, 041502 (2016).

[6] G. Deak, K. Curran, and J. Condell, Comput. Netw. 35, 1939 (2012).

[7] J. Xiao, Z. Zhou, Y. Yi, and L. M. Ni, ACM Comput. Surv. 49, 25 (2016).

[8] S. Palipana, B. Pietropaoli, and D. Pesch, Ad Hoc Netw. 64, 80 (2017).

[9] E. Aguirre, P. Lopez-Iturri, L. Azpilicueta, J. J. Astrain, J. Villadangos, D. Santesteban, and F. Falcone, Sensors 16, 1384 (2016).

[10] G. Chardon and L. Daudet, in Proceedings of the 2012 IEEE International Conference on Acoustics, Speech and Signal Processing (ICASSP) (IEEE, New York, 2012), pp. 9-12, https://ieeexplore.ieee.org/document/6287804/.

[11] G. Chardon, T. Nowakowski, J. de Rosny, and L. Daudet, IEEE J. Sel. Top. Signal Process. 9, 815 (2015).

[12] T. Nowakowski, J. de Rosny, and L. Daudet, J. Acoust. Soc. Am. 141, 2375 (2017).

[13] R. K. Ing, N. Quieffin, S. Catheline, and M. Fink, Appl. Phys. Lett. 87, 204104 (2005).

[14] L. Carin, D. Liu, and B. Guo, Inverse Probl. 24, 015023 (2008).

[15] Y. Jin, N. O’Donoughue, and J. M. F. Moura, in Proceedings of the 2008 IEEE International Conference on Acoustics, Speech and Signal Processing, ICASSP 2008 (IEEE, New York, 2008), pp. 3001-3004, https://ieeexplore.ieee .org/abstract/document/4518281/.

[16] Z.-H. Wu, Y. Han, Y. Chen, and K. J. R. Liu, IEEE Trans. Veh. Technol. 64, 1331 (2015).

[17] C. Chen, Y. Chen, Y. Han, H.-Q. Lai, and K. J. R. Liu, IEEE Internet Things J. 4, 111 (2017).

[18] M. Fink, Phys. Today 50, 34 (1997).

[19] G. Lerosey, J. de Rosny, A. Tourin, A. Derode, G. Montaldo, and M. Fink, Phys. Rev. Lett. 92, 193904 (2004).

[20] S. Sen, B. Radunovic, R. R. Choudhury, and T. Minka, in Proceedings of the 10th International Conference on Mobile Systems, Applications, and Services (ACM, New York, 2012), pp. 183-196, https://dl.acm.org/citation .cfm?doid=2307636.2307654.

[21] S. He and S.-H. G. Chan, IEEE Commun. Surveys Tutor. 18, 466 (2016).

[22] Y. Xie, T.-H. Tsai, A. Konneker, B.-I. Popa, D. J. Brady, and S. A. Cummer, Proc. Natl. Acad. Sci. U.S.A. 112, 10595 (2015).

[23] I. M. Vellekoop and A. P. Mosk, Opt. Lett. 32, 2309 (2007).

[24] S. M. Popoff, G. Lerosey, R. Carminati, M. Fink, A. Boccara, and S. Gigan, Phys. Rev. Lett. 104, 100601 (2010).

[25] A. P. Mosk, A. Lagendijk, G. Lerosey, and M. Fink, Nat. Photonics 6, 283 (2012).

[26] S. Rotter and S. Gigan, Rev. Mod. Phys. 89, 015005 (2017).

[27] M. Dupré, P. del Hougne, M. Fink, F. Lemoult, and G. Lerosey, Phys. Rev. Lett. 115, 017701 (2015). 
[28] P. del Hougne, F. Lemoult, M. Fink, and G. Lerosey, Phys. Rev. Lett. 117, 134302 (2016).

[29] N. Kaina, M. Dupré, G. Lerosey, and M. Fink, Sci. Rep. 4, 6693 (2014).

[30] P. del Hougne, B. Rajaei, L. Daudet, and G. Lerosey, Opt. Express 24, 18631 (2016).

[31] T. Sleasman, M. F. Imani, J. N. Gollub, and D. R. Smith, Phys. Rev. Applied 6, 054019 (2016).

[32] P. del Hougne, M. Fink, and G. Lerosey, Phys. Rev. Applied 8, 061001 (2017).

[33] J. Xiao, K. Wu, Y. Yi, L. Wang, and L. M. Ni, in Proceedings of the 2013 IEEE 33rd International Conference on Distributed Computing Systems (ICDCS) (IEEE, New York, 2013), pp. 236-245, https://ieeexplore .ieee.org/document/6681593/.

[34] A. Popleteev, in Proceedings of the 2013 ACM Conference on Pervasive and Ubiquitous Computing Adjunct Publication (ACM, New York, 2013), pp. 549-552, https://dl.acm .org/citation.cfm?doid=2494091.2497613.

[35] J. de Rosny and P. Roux, J. Acoust. Soc. Am. 109, 2587 (2001).

[36] J. de Rosny, P. Roux, M. Fink, and J. H. Page, Phys. Rev. Lett. 90, 094302 (2003).

[37] G. Maret, Curr. Opin. Colloid Interface Sci. 2, 251 (1997).

[38] T. D. Gould, Mood and Anxiety Related Phenotypes in Mice (Humana Press, New York, 2011).

[39] S. G. Conti, P. Roux, D. A. Demer, and J. de Rosny, Appl. Phys. Lett. 84, 819 (2004).

[40] Unraveling scattering and reflection contributions as in Eq. (1) becomes useful to localize multiple objects, otherwise, it is redundant and one could even work with intensity-only data.

[41] J. de Rosny, C. Debever, S. Conti, and P. Roux, Appl. Phys. Lett. 87, 154104 (2005).

[42] I. Dokmanić and M. Vetterli, in Proceedings of the 2 012 IEEE International Conference on Acoustics, Speech and Signal Processing (ICASSP) (IEEE, New York, 2012), pp. 2617-2620, https://ieeexplore.ieee.org/document/628 $8453 /$.

[43] B. Xiao, T. M. Antonsen, E. Ott, and S. M. Anlage, Phys. Rev. E 93, 052205 (2016).

[44] G. Gradoni, S. C. Creagh, G. Tanner, C. Smartt, and D. W. P. Thomas, New J. Phys. 17, 093027 (2015).

[45] A. Derode, A. Tourin, and M. Fink, Phys. Rev. E 64, 036606 (2001).

[46] F. Lemoult, G. Lerosey, J. de Rosny, and M. Fink, Phys. Rev. Lett. 103, 173902 (2009).

[47] M. Asefi and J. LoVetri, IEEE Trans. Microwave Theory Tech. 65, 3172 (2017).

[48] M. Asefi, A. Baran, and J. LoVetri, in Proceedings of the XXXIInd International Union of Radio Science General Assembly and Scientific Symposium (U.R.S.I., Ghent (Belgium), 2017), http://www.ursi.org/proceedings/procGA17/ papers/Paper_B38-2(2152).pdf.
[49] T. Sleasman, M. Boyarsky, M. F. Imani, T. Fromenteze, J. N. Gollub, and D. R. Smith, J. Opt. Soc. Am. B 34, 1713 (2017).

[50] P. del Hougne, M. F. Imani, T. Sleasman, J. N. Gollub, M. Fink, G. Lerosey, and D. R. Smith, Sci. Rep. 8, 6536 (2018).

[51] N. Kaina, M. Dupré, M. Fink, and G. Lerosey, Opt. Express 22, 18881 (2014).

[52] P. So, S. M. Anlage, E. Ott, and R. N. Oerter, Phys. Rev. Lett. 74, 2662 (1995).

[53] S. Hemmady, X. Zheng, T. M. Antonsen, Jr., E. Ott, and S. M. Anlage, Phys. Rev. E 71, 056215 (2005).

[54] U. Kuhl, H.-J. Stöckmann, and R. Weaver, J. Phys. A 38, 10433 (2005).

[55] J. Barthélemy, O. Legrand, and F. Mortessagne, Phys. Rev. E 71, 016205 (2005).

[56] See Supplemental Material at http://link.aps.org/ supplemental/10.1103/PhysRevLett.121.063901 for additional details on the experimental setup, localization procedure, toy model and considerations on real-life implementations.

[57] Y. C. Eldar and G. Kutyniok, Compressed Sensing: Theory and Applications (Cambridge University Press, Cambridge, England, 2012).

[58] J. M. Bioucas-Dias and M. A. T. Figueiredo, IEEE Trans. Image Process. 16, 2992 (2007).

[59] O. Katz, Y. Bromberg, and Y. Silberberg, Appl. Phys. Lett. 95, 131110 (2009).

[60] J. Hunt, T. Driscoll, A. Mrozack, G. Lipworth, M. Reynolds, D. Brady, and D. R. Smith, Science 339, 310 (2013).

[61] J. Hunt, J. Gollub, T. Driscoll, G. Lipworth, A. Mrozack, M. S. Reynolds, D. J. Brady, and D. R. Smith, J. Opt. Soc. Am. A 31, 2109 (2014).

[62] C. M. Watts, D. Shrekenhamer, J. Montoya, G. Lipworth, J. Hunt, T. Sleasman, S. Krishna, D. R. Smith, and W. J. Padilla, Nat. Photonics 8, 605 (2014).

[63] A. Liutkus, D. Martina, S. Popoff, G. Chardon, O. Katz, G. Lerosey, S. Gigan, L. Daudet, and I. Carron, Sci. Rep. 4, 5552 (2014).

[64] L. Atzori, A. Iera, and G. Morabito, Comput. Netw. 54, 2787 (2010).

[65] I. Goodfellow, Y. Bengio, and A. Courville, Deep Learning (MIT Press, Cambridge, MA, 2016).

[66] G. Satat, M. Tancik, O. Gupta, B. Heshmat, and R. Raskar, Opt. Express 25, 17466 (2017).

[67] V. Liu, A. Parks, V. Talla, S. Gollakota, D. Wetherall, and J. R. Smith, ACM SIGCOMM Comput. Commun. Rev. 43, 39 (2013).

[68] J. N. Gollub, O. Yurduseven, K. P. Trofatter, D. Arnitz, M. F. Imani, T. Sleasman, M. Boyarsky, A. Rose, A. Pedross-Engel, H. Odabasi et al., Sci. Rep. 7, 42650 (2017).

[69] P. del Hougne, M. F. Imani, M. Fink, D. R. Smith, and G. Lerosey, Zenodo (2018), DOI: 10.5281/zenodo.1217502. 